\title{
O dispositivo da especificidade matemática e a produção do sujeito-professor(a)-de-matemática
}

\section{The mathematical specificity device and the production of the subject-teacher-of- mathematics}

\author{
Jaqueline de Souza Pereira Grilo ${ }^{1}$ \\ Jonei Cerqueira Barbosa ${ }^{2}$ \\ Marlécio Maknamara ${ }^{3}$
}

\begin{abstract}
Resumo
Trata-se de um ensaio teórico que tem como objetivo discutir o dispositivo da especificidade matemática operado por professores de matemática. A construção da nossa argumentação apoiou-se em conceitos da caixa de ferramentas foucaultiana e incidiu sobre estudos que tratam do Conhecimento Matemático para o Ensino, do Conhecimento Especializado do Professor de Matemática e da Matemática para o Ensino. Problematizar o discurso da Matemática específica para ensinar possibilitou identificar a principal linha de força do dispositivo: a existência de uma Matemática específica para ensinar. Esta, por sua vez, emaranhada na rede que constitui o dispositivo produzem formas de ser-professor(a)-de-Matemática, dentro do jogo das relações de poder que pretende conduzir a conduta desses professores.
\end{abstract}

Palavras-chave: Dispositivo; Discurso; Ensino; Matemática.

\begin{abstract}
This theoretical essay aims to discuss the mathematical specificity device operated by mathematics teachers. The construction of our argument was based on concepts from the foucaultian toolbox and focused on studies dealing with Mathematical Knowledge for Teaching, the Mathematics Teacher's Specialized Knowledge of the and Mathematics for Teaching. To problematize the discourse of specific mathematics to teach made it possible to identify the main strength line of the device: the existence of specific mathematics to teach. This, in turn, entangled in the network that constitutes the device produces forms of being-a-teacher-of-Mathematics, within the game of power relations that intends to guide the conduct of these teachers.
\end{abstract}

Keywords: Device; Discourse; Teaching; Mathematics.

\section{Introdução}

Neste artigo, apresentamos um estudo que problematizou a rede de relações que pode ser estabelecida entre discursos que tratam de uma Matemática específica para ensinar e

\footnotetext{
Submetido em: 30/10/2020 - Aceito em: 01/02/2021 - Publicado em: 29/05/2021

${ }^{1}$ Doutora em Educação pela Universidade Federal da Bahia. Professor da Universidade Estadual de Feira de Santana, Brasil. E-mail: jspgrilo@uefs.br. ORCID: https://orcid.org/0000-0002-0408-047X

${ }^{2}$ Doutor em Educação Matemática pela Universidade Estadual Paulista Júlio de Mesquita Filho. Professor da Universidade Federal da Bahia, Brasil. E-mail: jonei.cerqueira@ufba.br. ORCID: https://orcid.org/0000-0002$\underline{4072-6442}$

3 Doutor em Educação pela Universidade Federal de Minas Gerais. Professor da Universidade Federal da Paraíba, Brasil. E-mail: maknamara@pq.cnpq.br. ORCID: https://orcid.org/0000-0003-0424-5657
} 
diferentes elementos que a viabiliza, tais como: documentos, instituições, leis, enunciados científicos, dentre outros. Aqui compreendemos discurso com base nos estudos de $\mathrm{M}$. Foucault, para o qual a compreensão extrapola o uso de signos para designar as coisas, ao tratá-lo como "práticas que formam sistematicamente os objetos de que falam" (Foucault, 2016, p. 60).

Esta compreensão sobre o que é discurso nos permite, por exemplo, ao ouvirmos a palavra maçã, associá-la à fruta, mas, também, ao pecado ou a um gesto de agrado a um(a) professor(a). Embora não negue que os discursos sejam feitos de signos, Foucault (2016, p. 60) destaca que eles fazem mais que utilizar signos para designar coisas, tornando-os "irredutíveis à língua e ao ato de fala". Na análise de discursos, é "esse 'mais' que é preciso fazer aparecer e que é preciso descrever" (Foucault, 2016, p. 60).

A essa rede de relações que se estabelece entre "discursos, instituições, organizações arquitetônicas, decisões regulamentares, leis, medidas administrativas, enunciados científicos, proposições filosóficas, morais, filantrópicas", Foucault (1989, p. 138) chamou de dispositivo. Um dispositivo, segundo Foucault (1989), visa não apenas constituir o sujeito, mas o aumento constante de sua utilidade.

A noção de sujeito expressa por M. Foucault, abandona a noção de "sujeito desde sempre aí" (Veiga-Neto, 2016, p. 107) e trabalha para demonstrar de que maneiras esse sujeito se constitui. Para Foucault (2006), não existe um sujeito preexistente ao discurso, o sujeito é constituído historicamente e culturalmente no seu tempo à medida que os discursos os atravessam. É, portanto, uma forma suscetível de se transformar, forma essa que nem sempre é idêntica a si mesma.

Estando o sujeito imerso na rede de relações que se identifica como dispositivo, sua produção não ocorre fora das relações de poder e saber. Assim, "o dispositivo tem sempre uma função estratégica concreta e se inscreve sempre em uma relação de poder" (Agamben, 2005, p. 10). Para Foucault (1999; 2014), o poder não se detém, só se exerce; ele está em toda parte como uma rede, uma relação de forças que atravessa os sujeitos.

Por mobilizar a perspectiva foucaultiana, a pesquisa insere-se no que se convencionou chamar de pós-estruturalismo, e tem o propósito de problematizar, analisar e desconstruir verdades singulares e contextuais que circunscrevem o nosso objeto de estudo. Entretanto, essa desconstrução não deve ser associada à destruição, mas a possibilidade de desmontar e remontar, de decompor e recompor o já dito (Paraíso, 2014). Apesar de não negar os procedimentos de pesquisa existentes, os pesquisadores pós-estruturalistas preferem não ficar presos a eles. Em alusão à metáfora do andarilho, o pesquisador percorre o caminho da pesquisa sem princípios rígidos, confrontando várias perspectivas (Tedeschi \& Pavan, 2017). Assim, desenvolvemos um ensaio teórico, que se caracteriza por apresentar uma argumentação lógica, rigorosa, coerente e crítica sobre um determinado tema (Barbosa, 2018). Coerente com a perspectiva pós-estruturalista, o nosso ensaio buscou problematizar os discursos, gerar desconfortos, não se restringindo a instaurar explicações e/ou novas teorizações sobre o tema. 
DOI: 10.20396/zet.v29i00.8661833

Em um ensaio teórico, o pesquisador não recorre a um caminho metodológico explícito, uma vez que este é um caminho tácito. Portanto, não há uma delimitação prévia de corpus da literatura, como ocorre nos estudos de revisão de literatura e estado da arte, ficando a cargo do pesquisador mobilizar a bibliografia necessária para produzir uma argumentação. Apoiados em Fischer (2002, p. 43), a bibliografia mobilizada foi analisada "igualmente como práticas que são, como constituidores de sujeitos e corpos, de modos de existência não só de pessoas como de instituições e inclusive de formações sociais mais amplas”.

Nas seções a seguir, situamos como o discurso da Matemática específica para ensinar tem circulado nos estudas da área de Educação Matemática para, em seguida, discutirmos a rede de relações que se estabelece entre esse discurso e elementos diversos que nomeamos como dispositivo da especificidade Matemática.

\section{Discurso da Matemática específica para ensinar}

Ao enveredarmos pelo campo das pesquisas foucaultianas, buscamos fazer aparecer algo mais nos discursos que circulam nas investigações da área de Educação Matemática e que tratam de uma Matemática que seria específica para o ensino. Esses discursos têm circulado em estudos que adotam diferentes conceptualizações sobre o tema. Liping Ma, por exemplo, conceptualizou a noção de Profound Understanding of Fundamental Mathematics (PUFM), requerendo um(a) professor(a) que não apenas conhecesse a estrutura conceitual e as atitudes básicas da Matemática inerentes à matemática elementar, mas também que fosse capaz de ensiná-las aos alunos (Ma, 1999).

Contemporânea dos trabalhos de Liping Ma, a pesquisadora Deborah Ball, juntamente com seus colaboradores, desenvolve a noção de Mathematical Knowledge for Teaching $(M K T)$, um conhecimento matemático específico para o ensino que seria diferente do conhecimento matemático necessário a outros profissionais como um engenheiro, por exemplo (Ball \& Bass, 2003). Estes pesquisadores e pesquisadoras, inspirados nos estudos de Lee Shulman sobre o domínio do conhecimento profissional do professor (Shulman, 1987), dedicam esforços para desenvolver a noção de uma Matemática específica para o ensino e inspiraram muitos outros estudos na área. Para darmos uma ideia da variabilidade dessas conceptualizações, citamos o trabalho Neubrand (2018) no qual o autor discute três delas: Mathematics for Teaching (MfT), Knowledge Quartet (KQ) e Structure Model (SM).

Dada a diversidade de conceptualizações, voltamos a nossa análise sobre aquelas que têm configurado em boa parte das pesquisas que tratam do tema no Brasil: Mathematical Knowledge for Teaching (MKT), que traduzimos como Conhecimento Matemático para o Ensino (CME); o Mathematics Teacher's Specialized Knowledge (MTSK), traduzido como Conhecimento Especializado do Professor de Matemática (CEPM); e a Mathematics for Teaching $(M f T)$ ou, no português, Matemática para o Ensino (MpE). Assim, usamos a expressão "discurso da Matemática específica para ensinar" na tentativa de capturar o discurso que contém o CME, o CEPM e a MpE.

As investigações realizadas sob a perspectiva do CME o tomam como uma teoria que 
seria capaz de mapear o conhecimento matemático necessário para executar as tarefas recorrentes no ensino de Matemática, principalmente após o estudo desenvolvido por Ball, Thames \& Phelps (2008). Nesse estudo, os autores apresentam uma nova categorização sobre o Conhecimento do Conteúdo e o Conhecimento Pedagógico do Conteúdo elaborados por Lee Shulman e identificam subdomínios para cada um deles amplamente divulgados em pesquisas da área, tanto nacional quanto internacionalmente (Ribeiro, 2012; Kim, 2016; Speer, King \& Howell, 2015).

A busca por esse refinamento gerou outras frentes de estudo das quais destacamos o estudo de José Carrillo e colaboradores que reelaboram os domínios do CME ao propor que o conhecimento de todos os professores e professoras é especializado, configurando a noção de Conhecimento Especializado de Professores de Matemática (CEPM) (Carrillo, Climent, Contreras \& Muñoz-Catalán, 2013). O CEPM também se organiza em domínios e subdomínios que focalizariam demandas relacionadas, especificamente, ao ensino de Matemática. Junto a essa perspectiva, o grupo liderado pelo professor Miguel Ribeiro tem defendido que a prática letiva de professores de/que ensinam Matemática deveria apoiar-se em um conhecimento matemático amplo e profundo afim de orientar os(as) estudantes na construção do conhecimento a partir de seus próprios raciocínios e produções, valorizando os processos não padronizados ou incorretos como oportunidade de aprendizagem (Di Martino, Mellone \& Ribeiro, 2019). No Brasil, o CEPM tem sido amplamente divulgado pelo professor Miguel Ribeiro sob a ótica do Conhecimento Especializado e Interpretativo do professor de/que ensina Matemática (Ribeiro, Mellone \& Jakobsen, 2016; Ribeiro, Policastro, Almeida, Caldatto \& Mellone, 2018).

Outrossim, há investigações que adotam uma perspectiva discursiva sobre a Matemática específica para ensinar argumentando que o conhecimento sobre a Matemática é inseparável daquele estabelecido na prática docente (Davis \& Simmt, 2006). Segundo os autores, essas investigações não visam apenas identificar o que é ou o que os(as) professores sabem sobre Matemática, mas contribui para a produção de novas possibilidades interpretativas ao propor novas formas de explorá-lo. Buscam apontar o que seria a natureza complexa do fenômeno, evitando configurá-lo por meio de domínios, o que, para esses pesquisadores, seriam tentativas reducionistas de descrição do mesmo (Davis \& Simmt, 2006; Davis \& Renert, 2014). Esses estudos acreditam ir além da análise do domínio do(a) professor(a) sobre o conteúdo matemático ao focalizarem como esses constroem discursivamente o conhecimento matemático para ensinar (Barwell, 2013). A esses estudos tem sido associada a ideia de MpE. Para os autores, a MpE está distribuída no coletivo de professores, de modo que o conhecimento individual e coletivo não podem ser dicotomizado.

À luz de uma perspectiva foucaultiana, dizemos que a variabilidade de conceptualização sobre o discurso da Matemática específica para ensinar expressa a disputa para impor significados sobre o ensino de Matemática, o que termina por constituir determinados tipos de sujeito-professor(a)-de-Matemática. Com base em Grilo, Barbosa e Maknamara (2020), consideramos que o sujeito-professor(a)-de-Matemática é o sujeito "desejado" pelo discurso da Matemática específica para ensinar, aquele que foi subjetivado, 
que se identifica ou é identificado por discursos do CME, CPEM e MpE.

Investigar de que modo vem se dando a constituição desse sujeito, uma "analítica do sujeito" nas palavras de Veiga-Neto (2016), requer examinar os discursos que o envolvem e o constituem. Segundo M. Foucault, essa produção do sujeito não ocorre fora das relações de poder.

O poder, acho eu, deve ser analisado como uma coisa que circula, ou melhor como uma coisa que só funciona em cadeia. Jamais ele está localizado aqui ou ali, jamais está entre as mãos de alguns, jamais é apossado como uma riqueza e um bem. $\mathrm{O}$ poder funciona. $\mathrm{O}$ poder se exerce em rede e, nessa rede, não só os indivíduos circulam, mas estão sempre em posição de ser submetidos a esse poder e também de exercê-lo. Jamais eles são o alvo inerte ou consentidor do poder, são sempre seus intermediários. (Foucault, 1999, p. 35).

Decorre, portanto, o entendimento de que o discurso da Matemática específica para ensinar coloca relações de poder em funcionamento para conduzir a conduta de professores e professoras de Matemática, no sentido de se deixar guiar por outrem, mas também de se auto guiar. Nas palavras de Foucault (2008, p. 255), "a conduta é, de fato, a atividade que consiste em conduzir, a condução, se quiserem, mas é também a maneira como uma pessoa se conduz, a maneira como se deixa conduzir, a maneira como é conduzida".

Segundo Foucault (1989), não existe verdade fora das relações de poder; além disso, ela produz e é produzida por efeitos regulamentadores de poder. Assim, o que há são regimes de verdade, ou seja, discursos que são acolhidos e passam a funcionar como verdadeiros. Assim, o discurso da Matemática específica para ensinar busca imprimir regimes de verdade sobre como ver o saber dos professores e, por consequência, como formá-los e como ensinar Matemática. Conforme Foucault (1989), há mecanismos e instâncias habilitadas/reconhecidas como capazes de distinguir e sancionar discursos verdadeiros ou falsos; há técnicas e procedimentos valorizados para a obtenção da verdade, como o método científico, por exemplo; e há aqueles que têm a função de dizer o que funciona como verdadeiro.

As investigações (Ball, Thames \& Phelps, 2008; Tatto, 2013; Carrillo, Climent, Contreras \& Muñoz-Catalán, 2013; Davis \& Renert, 2014; Phelps \& Howell, 2016; Ribeiro, Mellone \& Jakobsen, 2016; Santos \& Barbosa, 2016), e os enunciados científicos a elas correlatos, que fazem circular o discurso da Matemática específica para ensinar, são associadas a instituições de pesquisa, ocorrem em organizações arquitetônicas (escolas e universidades) e estão atentas às legislações curriculares. Por meio de métodos científicos, com financiamento público ou privado, buscam aperfeiçoar cada vez mais seus instrumentos de pesquisa para manterem o seu encargo de dizer o que seria a "verdade" sobre a Matemática específica para ensinar. Assim, dizemos que o discurso da Matemática específica para ensinar, dotado de uma racionalidade estratégica, se engendra em torno de um dispositivo que dispõe os discursos de modo a constituir o sujeito-professor(a)-deMatemática. 


\section{Dispositivo da especificidade matemática}

De acordo com Agamben (2005), o dispositivo dispõe uma série de práticas (discursivas e não-discursivas) e mecanismos (jurídicos, técnicos, científicos, militares) com o objetivo de fazer frente a uma urgência e de obter um efeito. Viabilizado por um grupo heterogêneo de elementos, "um dispositivo dispõe algo em uma organização peculiar, dentro de uma racionalidade particular" (Maknamara, 2011, p. 69). No caso do dispositivo que nomeamos como dispositivo da especificidade matemática, dentre esses elementos destacamos: o discurso da Matemática específica para ensinar, as organizações de pesquisas e seus enunciados científicos, as decisões regulamentares (especialmente aquelas que se referem a diretrizes curriculares) e as organizações envolvidas (universidades e escolas). Se todo dispositivo "é uma rede cuja composição trabalha a favor de efeitos específicos de poder" (Maknamara, 2011, p. 129), diremos que esse dispositivo tem o objetivo de conduzir a conduta de professores(as) de/que ensinam Matemática, no sentido torná-los úteis, capacitados para a boa docência de Matemática.

Para ilustrar como esses discursos abordam a boa docência de Matemática, destacaremos trechos de estudos seminais sobre o CME, CPEM e MpE. Primeiramente, os estudos apontam que capacitar os(as) professores(as) de/que ensinam Matemática para a boa docência envolve o descompactar o conhecimento matemático, pois acreditam que apresentar aos(às) estudantes aquilo que geralmente é comprimido no fazer matemático, pode tornar esse conhecimento mais acessível.

(...) looking at teaching as mathematical work highlights some essential features of knowing mathematics for teaching. One such feature is that mathematical knowledge needs to be unpacked. This may be a distinctive feature of knowledge for teaching. Consider, in contrast, that a powerful characteristic of mathematics is its capacity to compress information into abstract and highly usable forms. (Ball \& Bass, 2003, p. 11)

Teaching involves the use of decompressed mathematical knowledge that might be taught directly to students as they develop understanding. (Ball, Thames \& Phelps, 2008, p. 400)

Esses discursos apontam que a boa docência em Matemática não se resume a descompactar o conhecimento matemático, mas também requer que os(as) professores(as) desenvolvam a capacidade de articular diferentes áreas da Matemática, bem como diferentes níveis do conhecimento.

Another important aspect of knowledge for teaching is its connectedness, both across mathematical domains at a given level, and across time as mathematical ideas develop and extend. Teaching requires teachers to help students connect ideas they are learning-geometry to arithmetic, for example. (Ball \& Bass, 2003, p. 11)

Teaching also requires teachers to anticipate how mathematical ideas change and grow. Teachers need to have their eye on students" "mathematical horizons" even as they unpack the details of the ideas in focus at the moment (Ball, 1993). For example, second grade teachers may need to be aware of the fact that saying, "You can't subtract a larger number from a smaller one", is to say something that, although pragmatic 
when teaching whole number subtraction, is soon to be false. (Ball \& Bass, 2003, p. 12)

Além dessa articulação, também é esperado que os(as) professores(as) consigam bem reger a sua aula. Essa regência perpassa por saber escolher as melhores estratégias de apresentação do conhecimento matemático, as formas de articulação entre perguntas e respostas, entre avançar ou adiar a apresentação de um novo conteúdo, por exemplo.

During a classroom discussion, a teacher must decide when to pause for more clarification, when to use a student's remark to make a mathematical point, and when to ask a new question or pose a new task to further students' learning. Each of these decisions requires coordination between the mathematics at stake and the instructional options and purposes at play. (Ball, Thames \& Phelps, 2008, p. 401)

Não se espera, contudo, que estas capacidades estejam dissociadas de uma compreensão sobre diferentes modos de se fazer matemática. Nesse sentido, é requerido que o(a) professor(a) de Matemática tenha a capacidade de interpretar diferentes métodos e soluções apresentadas aos problemas.

Knowing mathematics for teaching often entails making sense of methods and solutions different from one's own, and so learning to size up other methods, determine their adequacy, and compare them, is an essential mathematical skill for teaching, and opportunities to engage in such analytic and comparative work is likely to be useful for teachers. (Ball \& Bass, 2003, p. 13)

Significant to this example is that a teacher's own ability to solve a mathematical problem of multiplication ( $35 \times 25)$ is not sufficient to solve the mathematical problem of teaching - to inspect alternative methods, examine their mathematical structure and principles, and to judge whether or not they can be generalized. (Ball \& Bass, 2003, p. 7).

A capacidade de compreender métodos não padronizados de solução de um problema, ganha potência nesses discursos quando destacam que também cabe ao professor(a) a capacidade de interpretar métodos incorretos realizados pelos estudantes.

Recognizing that this student's answer as wrong is one step, to be sure. But effective teaching also entails analyzing the source of the error. (Ball \& Bass, 2003, p. 17)

However, teaching involves more than identifying an incorrect answer. Skillful teaching requires being able to size up the source of a mathematical error. Moreover, this is work that teachers must do rapidly, often on the fly, because in a classroom, students cannot wait as a teacher puzzles over the mathematics himself. (Ball, Thames \& Phelps, 2008, p. 397)

This interpretative knowledge, being part of mathematical knowledge, is defined as the knowledge that allows teachers to give sense to pupils' non-standard answers (i.e., adequate answers that differ from the ones teachers would give or expect) or to answers containing errors. (Ribeiro, Mellone \& Jakobsen, 2016, p. 9)

Para esses discursos, essas e outras capacidades requeridas ao(a) professor(a) que ensina Matemática, não ocorrem fora de um dado contexto. Neste sentido, o conhecimento matemático mobilizado pelos(as) professores(as) deveria levar em consideração o contexto social e interacional no qual se desenvolve. 
DOI: $10.20396 /$ zet.v29i00.8661833

(...) since human interaction is fundamentally discursive, any accounts of knowing, meaning, intending and so on are inevitably shaped by the immediate temporal, social and interactional context in which they arise. (Barwell, 2013, p. 600)

Because of its dynamic and nested character, mathematics-for-teaching cannot be considered a domain of knowledge to be mastered by individuals. It always occurs in contexts that involve others - and, hence, an awareness of how others might be engaged in productive collectivity is an important aspect. It is thus that our research into teacher's mathematics-for-teaching is oriented by an assumption that we also take into our teaching of mathematics: The 'learning system' that the teacher can most directly influence is not the individual student, but the classroom collective (Davis and Simmt, 2003). (Davis \& Simmt, 2006, p. 309)

Face ao exposto, compreendemos que o dispositivo da especificidade matemática é mais um tipo de dispositivo pedagógico, ou seja, um dispositivo que "cuida da aprendizagem calculada dos efeitos desejados no âmbito de uma racionalidade (...) que ensina indivíduos a tornarem-se sujeitos dotados daquilo que o poder quer" (Maknamara, 2020, p. 144). O dispositivo da especificidade matemática tem a formação de professores(as) que ensinam Matemática como campo privilegiado de atualização e como principal linha de força a defesa de que existiria um conhecimento matemático específico para ensinar. Segundo Deleuze (1996), a linha de força passa por todos os lugares de um dispositivo, muitas vezes invisível e indizível, mas estreitamente emaranhada em outras linhas e totalmente desenredável. Os discursos do CME, do CEPM e da MpE são linhas que também se emaranham nessa rede que, em meio as relações de poder e saber, favorecem o aparecimento de novos modos de subjetivação.

Neste sentido, Agamben (2005, p. 13) diz que o sujeito é o "que resulta da relação e, por assim dizer, do corpo-a-corpo entre os viventes e os dispositivos". Ao olharmos para a produtividade advinda da rede de relações que se estabelece entre esses elementos, voltamonos para os modos de subjetivação, os tipos de sujeito-professor(a)-de-Matemática, demandados e constituídos peça por peça por este dispositivo. Corroborando Agamben (2005), em estudo anterior, argumentamos que o discurso da Matemática específica para ensinar tem se voltado a produzir sujeitos que devem ser capazes de: descompactar, conectar, articular, antecipar, compreender e provar ideias matemáticas de modo a torná-las mais acessíveis aos estudantes, por isso espera-se que também sejam sujeitos participativos e engajados em situações coletivas nas quais possam discutir e, consequentemente, aumentar ou reconfigurar o seu repertório conceitual. São sujeitos controlados pelo contexto no qual se inserem, conhecedores dos guias curriculares, exploradores de oportunidades e (re)formuladores de conceitos matemáticos (Grilo; Barbosa; Maknamara, 2020).

Deleuze (1996) afirma que na rede que emaranha os elementos heterogêneos constitutivos do dispositivo, saber, poder e subjetividade não apresentam contornos definidos, são vetores ou tensores que vão permitir o surgimento de novas linhas no dispositivo. Antes, porém, de tentar identificar que novas linhas seriam essas, procuraremos problematizar àquelas que surgem relacionadas com a dimensão das relações de poder.

Para ilustrar como o discurso da Matemática específica para ensinar mobiliza 
diferentes relações de poder, inicialmente destacamos como ele opera com o poder soberano, apresentando excertos de estudos que fazem circular os discursos do CME, CPEM e MpE. Em outro estudo, Grilo e Barbosa (2021) apresentam uma análise mais aprofundada sobre como o discurso da Matemática específica para ensinar mobiliza relações de poder para conduzir a conduta de professores(as).

We hypothesized that teachers' opportunities to learn mathematics for teaching could be better tuned if we could identify those types [de conhecimento] more clearly. If mathematical knowledge required for teaching is indeed multidimensional, then professional education could be organized to help teachers learn the range of knowledge and skill they need in focused ways. If, however, the mathematical knowledge required for teaching is basically the same as general mathematical ability, then discriminating professional learning opportunities would be unnecessary. Based on our analysis of the mathematical demands of teaching, we hypothesized that Shulman's content knowledge could be subdivided into CCK and specialized content knowledge and his pedagogical content knowledge could be divided into knowledge of content and students and knowledge of content and teaching. (Ball, Thames \& Phelps, 2008, p. 399)

In contrast, MTSK, by virtue of being designed to encapsulate teacher's specialized Knowledge, focuses its attention on mathematical content and, with greater precision, on the different ways of fully engaging with mathematical content when teaching. (Carrillo, Climent, Contreras \& Muñoz-Catalán, 2013)

Percebe-se que o poder soberano se mostra por meio do discurso da Matemática específica para ensinar quando este advoga para si a soberania de um campo de conhecimento específico, o qual os estudos desenvolvidos por Shulman (1987) não foi capaz de detectar. Esse saber ocupa o lugar do soberano que, objetivando defender a si mesmo ou ao seu território (as práticas de ensino de Matemática), detém o direito sobre a vida e a morte dos seus súditos (os(as) professores(as) de Matemática). Esse direito se exerce ao "fazer morrer" práticas docentes baseadas em uma Matemática que não é específica para o ensino, permitindo "deixar viver" uma prática docente que dê conta de tais especificidades.

A este poder soberano vê-se aninhado um poder pastoral que pretende guiar o seu rebanho (o conjunto de professores(as) que ensina Matemática) para a salvação, levando-os a transitar entre diferentes domínios de conhecimento, como proposto por Ball, Thames \& Phelps (2008) (Figura 1) e Carrillo, Climent, Contreras \& Muñoz-Catalán (2013) (Figura 2) ou diferentes ênfases conceituais como proposto por Davis \& Renert (2014) (Figura 3). 
DOI: 10.20396/zet.v29i00.8661833
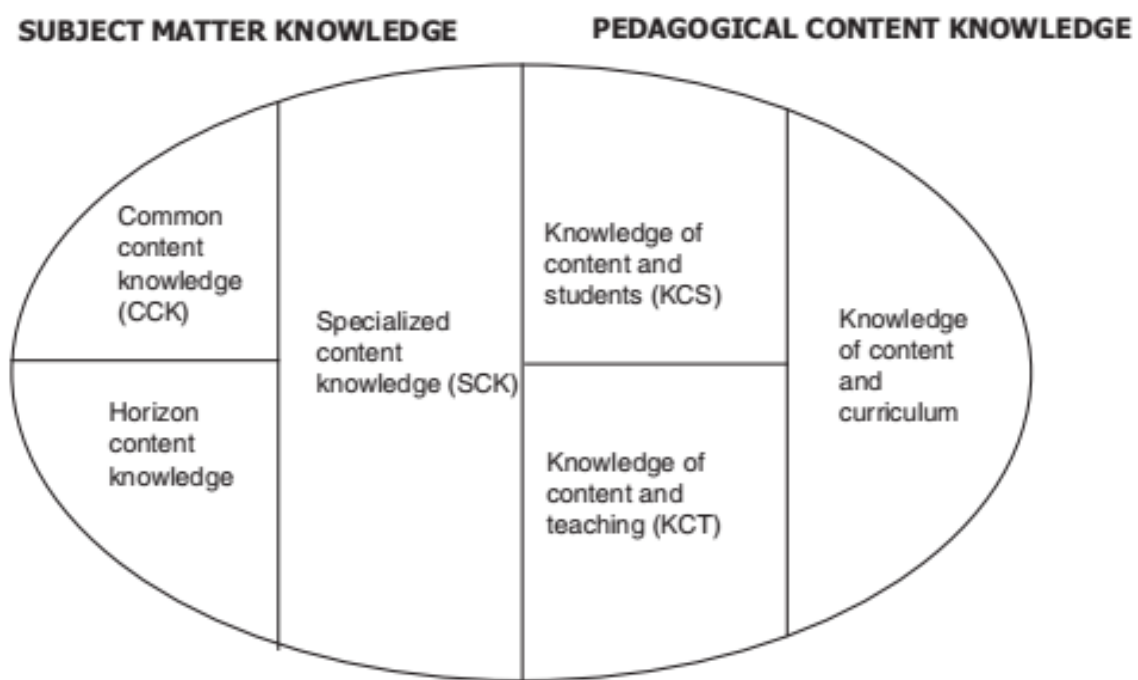

Figura 1. Domínios do Conhecimento Matemático para o Ensino Fonte: Ball, Thames e Phelps (2008, p. 403)

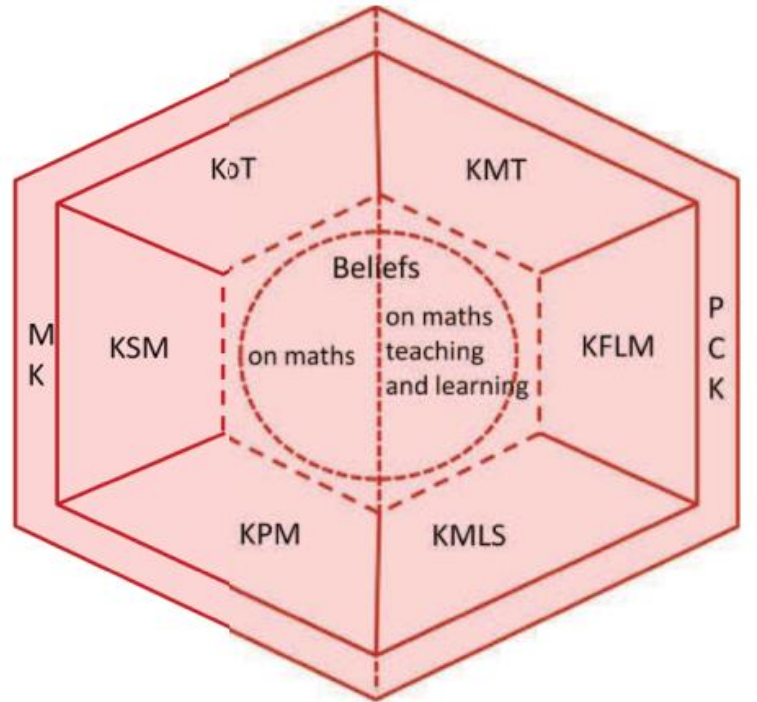

Figura 2. Gráfico do Conhecimento Especializado do Professor de Matemática Fonte: Carrillo, Climent, Contreras e Muñoz-Catalán (2013, p. 2989) 


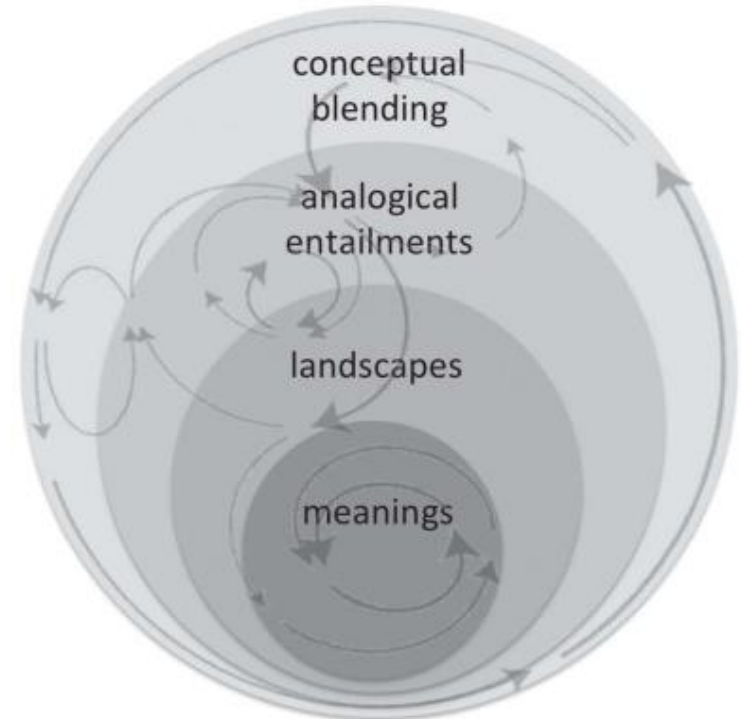

Figura 3. Uma metáfora visual para descrever as relações entre ênfases de estudo de conceito Fonte: Davis e Renert (2014, p. 57)

O acompanhamento desse transitar entre domínios e ênfases conceituais se dá pelo exame minucioso do coletivo de professores(as) que ensina Matemática realizado pela operação de um poder disciplinar. O poder disciplinar "toma os indivíduos ao mesmo tempo como objetos e como instrumentos de seu exercício" (Foucault, 2014, p. 167). Assim, os(as) professores(as) são examinados, bem como agem como instrumento que examina as práticas matemáticas de seus(suas) estudantes. O exame "é um controle normalizante, uma vigilância que permite qualificar, classificar e punir" (Foucault, 2014, p. 181).

Projetos como o Learning Mathematics for Teaching Project (LMT) (http://www.umich.edu/ lmtweb/) e o Teacher Education and Development Study in Mathematics (TEDS-M) (Tatoo, 2013) utilizam a técnica do exame por meio da elaboração, aplicação e refinamento de instrumentos avaliativos aplicados em larga escala. Estes projetos visam, dentre outros fins: um controle minucioso da estrutura do conhecimento matemático do professor (Ní Ríordáin, Paolucci \& O'Dwyer, 2017); relacioná-los com o desempenho matemático dos estudantes (Delaney, 2012; Tchoshanov, 2011), refinar instrumentos de avaliação (Phelps, Kelcy, Jones \& Liu, 2016). Aliado a isso, vê-se crescendo na área o desenvolvimento de cursos de formação continuada de professores que visam examinar as suas práticas em torno do conhecimento mobilizado ao ensinar (Rangel, Giraldo \& Maculan Filho, 2015; Di Bernardo, Policastro, Almeida, Ribeiro, Melo \& Aiub, 2018), de como interpretam as produções de seus estudantes (Couto \& Ribeiro, 2017; Ribeiro, 2017), de como mobilizam diferentes representações de um dado conceito matemático (MenduniBortoloti \& Barbosa, 2018; Santos \& Barbosa, 2016).

Agindo de forma articulada e mutuamente sobre corpos múltiplos, esses poderes tornam-se ainda mais insidiosos ao pretender governar a vida do conjunto de professores(as) de Matemática quando entra em ação o biopoder. O biopoder, diferente do poder soberano e do poder disciplinar (mas não separado destes), não se exerce sobre um corpo, mas sobre um corpo múltiplo - a população (aqui compreendida como o coletivo de professores que 
ensinam Matemática) - por meio de regulamentações que buscam o governo da vida (Foucault, 1989). Não é difícil encontrarmos entre os estudos sobre o CME, o CEPM e a $\mathrm{MpE}$ indicações generalizantes para políticas públicas de formação de professores que pretendem promover um governo da vida e até sobre implicações do conhecimento matemático para o desenvolvimento de um país, como se vê a seguir.

Curricular implementations are unlikely to deliver the anticipated benefits for mathematics learners if written guidance to teachers is interpreted and enacted differently from the ways that policymakers and curriculum designers intend. (Foster \& Inglis, 2017, p. 2)

In the new economy, mathematics has emerged as the gateway discipline (...). Yet the sorts of mathematical competence that are of value to current and future society bear ever-diminishing resemblance to the emphases seen in contemporary classrooms.

(...) but in spite of large-scale initiatives to rethink the content of school mathematics (e.g., NCTM, 1989, 2000), so far, few educational institutions have demonstrated a capacity to match the pace of cultural change. (...) the fundamental content has changed very little from previous decades and even centuries. Many reasons can be (and have been) mentioned: social inertia, limited resourcing of schools, fatigue with ever-swinging reform pendula, systemic resistance to reform, and so on. We believe that a critical (if not the main) issue here is a lack of capacity in teachers to deal with new mathematical content, and even with current content, in compelling ways. In other words, the stagnation has much to do with teachers' disciplinary knowledge of mathematics.

(...)

Schools have traditionally emphasized the development of technical competence, which was an obvious need in an industrial economy. But in a knowledge-based economy, the development of conceptual fluency is of greater importance and has been the focus of major initiatives in school mathematics. Our research into the subtlety and complexity of teachers' knowledge not only reveals that some major shortcomings of these initiatives, it also offers an important possible route to achieving the goal of true conceptual fluency - a profound understanding of emergent mathematics. (Davis \& Renert, 2014, p. 13-127).

Como se pudéssemos pôr uma lupa sobre as linhas que se entrelaçam no dispositivo da especificidade matemática e, mesmo que por um segundo, pudéssemos capturá-lo, construímos uma rede ${ }^{4}$ (Figura 4) que entrelaça as relações de poder e saber e o que se demanda aos sujeitos que ensinam Matemática para que sejam reconhecidos e possam reconhecer-se como "bons professores(as)" de Matemática. Para isso, olhamos para aquilo que é demandado aos professores(as) de Matemática pelo discurso da Matemática específica para ensinar como um "ponto" na rede que configura, num dado momento histórico, o dispositivo da especificidade matemática, visto que eles definem a condição que possível de ser ocupada pelo sujeito-professor(a)-de-Matemática.

\footnotetext{
${ }^{4}$ Para a construção da rede foi utilizado o software Gephi.
} 
DOI: $10.20396 /$ zet.v29i00.8661833

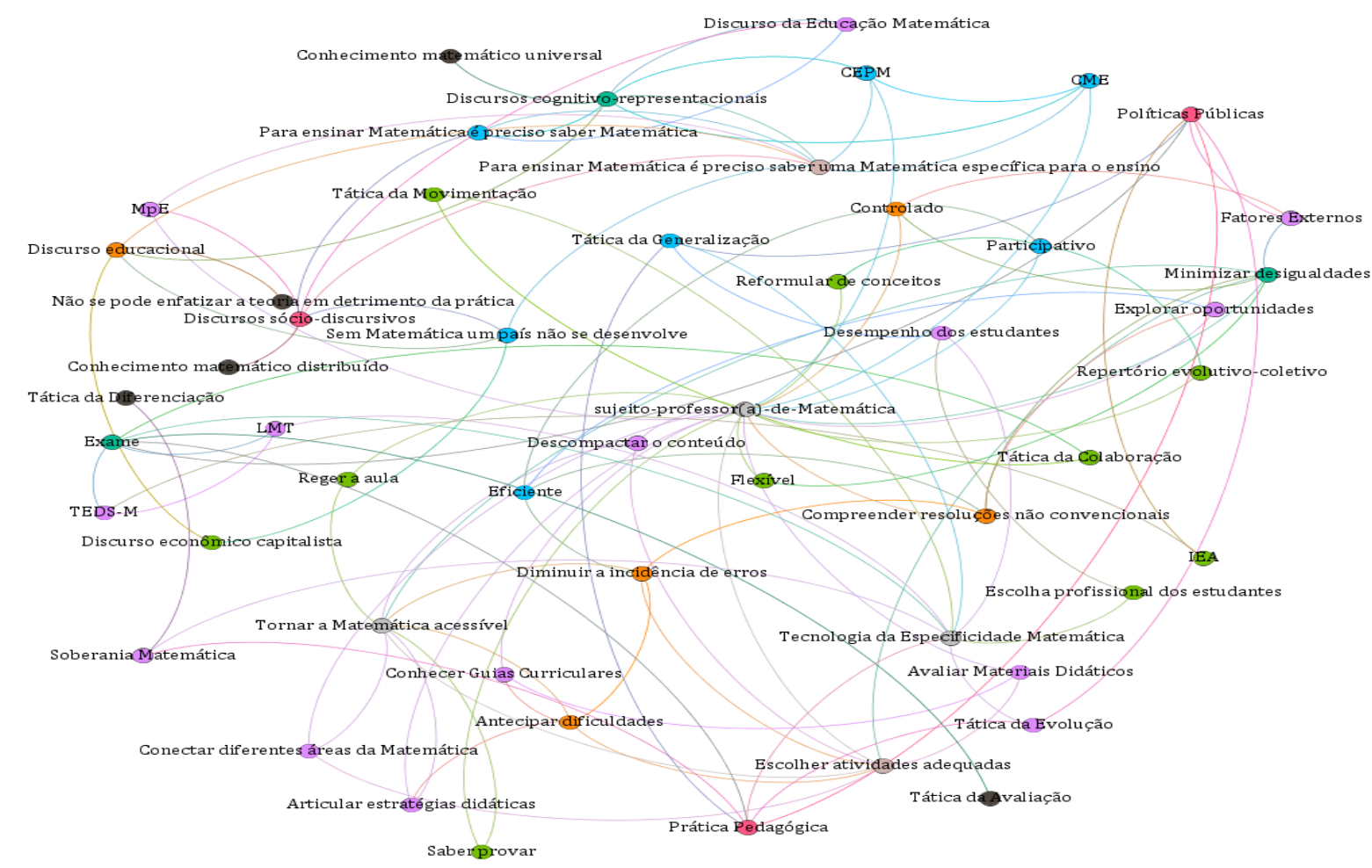

Figura 4. As relações de poder e saber e o que se demanda ao sujeito-professor(a)-de-Matemática Fonte: Os autores

O dispositivo da especificidade Matemática opera para tornar cada vez mais visíveis os(as) professores(as) que ensinam Matemática, emaranhando saber e poder. Como nos ensinou Foucault (1999), olhamos como o poder funciona e, ao capturar o seu funcionamento, descrevemos a rede que o emaranha, entrelaça e articula; a rede que o faz capilar, transitório, pulverizado e descentrado. Na descrição dessa rede, o sujeitoprofessor(a)-de-Matemática está em posição de ser submetido a esse poder, mas também de exercê-lo, pois “o indivíduo é um efeito do poder e é, ao mesmo tempo, na mesma medida em que é um efeito seu, seu intermediário: o poder transita pelo indivíduo que ele constituiu" (Foucault, 1999, p. 35). Dentre tantas linhas momentaneamente capturadas, vimos que o sujeito demandado pelo discurso da Matemática específica para ensinar concorre para "Tornar a matemática acessível aos estudantes", "Diminuir a incidência de erros" e "Identificar os fatores externos (ou internos) que influenciam na (não) aprendizagem da Matemática”.

\section{Considerações finais}

Compreendemos o dispositivo da especificidade matemática como a rede de relações de poder que emaranha diferentes campos de saber (Matemática, Pedagogia, Economia) afim de informar modos de ser professor(a) de Matemática útil para o desenvolvimento do seu país. Tomamos o poder como uma instância produtiva, fabricante de subjetividades. Desse modo, ao nos voltarmos para a rede que mostra as relações de poder e saber e os modos de subjetivação do professor(a) de Matemática, não buscamos exibir uma lei geral ou uma 
estrutura que revelasse como essas relações são exercidas, buscamos representar o quanto essas relações são complexas.

Envoltas a essa complexidade representada pela rede, as relações de poder e saber favorecem o aparecimento de novos modos de subjetivação, haja vista a proliferação discursiva que elas proporcionam. Vimos, por exemplo, que essa trama discursiva demanda um sujeito-professor(a)-de-Matemática que: torna a Matemática acessível aos estudantes; diminui a incidência de erros dos estudantes; é atento aos fatores externos a prática pedagógica; é responsável pelo desempenho dos estudantes em teste padronizados; é responsável por suas escolhas profissionais; submete-se ao exame, enquanto instrumento que escrutina a prática pedagógica para fornecer informações a Políticas Públicas; articula teoria e prática; e promove o desenvolvimento do seu país. Esses novos modos de subjetivação aparecem, especialmente, quando incidimos a análise sobre o discurso da Matemática específica para ensinar e o vimos se desdobrar em entrelaçamentos discursivos que não se restringem apenas ao campo da Educação Matemática, encontrando "pontos" no campo de discursos educacionais e econômicos.

O sujeito-professor(a)-de-Matemática ocupa na rede um lugar de destaque, não por ser o centro (não há centralidade), mas por ser ao mesmo tempo emissor e receptor de poder. Esse sujeito, enquanto posição que pode ser ocupada, resulta de uma construção discursiva que se dá no âmbito de regimes de verdades que se estabelecem em relação ao tempo e ao espaço. Não sendo esses regimes de verdade fixos, o que apresentamos neste ensaio é uma construção datada, própria do tempo em que foi escrita, e carregada daquilo que nos posiciona como sujeito-professor(a). Essa construção assinala que o discurso da Matemática específica para ensinar visa a conformar a soberania da Matemática como um saber que tem seus próprios regimes de verdade e que advoga para si uma prática discursiva já formalizada, como um "modelo para a maioria dos discursos científicos em seu esforço de alcançar o rigor formal e a demonstratividade" (Foucault, 2016, p. 228).

Ao reafirmar essa soberania, o discurso da Matemática específica para ensinar nega a possibilidade de outras Matemáticas adentrarem o ambiente escolar. Sobre este aspecto, não identificamos no discurso da Matemática específica para ensinar, domínios ou ênfases conceituais que tratassem de uma Matemática não-escolar. Também não identificamos que as adaptações de itens dos testes disponibilizados pelo projeto Learning Mathematics for Teaching (LMT) (Know, Thames \& Pang, 2012), ocorreram para o reconhecimento de outras Matemáticas. Do mesmo modo, negam a possibilidade de a Matemática perder a sua soberania frente a outro saber escolarizado, haja vista que quando domínios e ênfases tratam de conectar diferentes área ou realizar combinações conceituais, problematizam áreas da própria Matemática (por exemplo: Aritmética, Álgebra e Geometria) e não discutem modos de abordá-la de forma interdisciplinar. Por ser considerada soberana, a Matemática por si, basta!

Entretanto, esta afirmação não diz que esse discurso segue indiferente aos contextos que o cerca. Lembremos do estudo de Hoover, Mosvold, Ball \& Lai (2016), quando os 
DOI: $10.20396 /$ zet.v29i00.8661833

autores apontam que a área tem começado a abordar a persistente desigualdade da aprendizagem matemática produzida e reproduzida nas escolas. Contudo, ao olharmos para a trama discursiva representada pela rede, perguntamos se esse interesse da área visa a romper com a soberania da Matemática, dando lugar a outras Matemáticas e a outros campos de saber; ou visa a atender a um discurso econômico-capitalista para o qual, entre outras coisas, um país precisa da Matemática para se desenvolver?

Por hora, os entrelaçamentos discursivos que aparecem na rede nos mostram que a posição de sujeito que diz da necessidade de professores e professoras tornarem a Matemática acessível aos estudantes, se entrelaça com outros discursos que realizam um exame minucioso das práticas pedagógicas das quais participam, a fim de fornecer subsídios às políticas públicas educacionais. As políticas públicas educacionais, segundo Stephen Ball, estão cada vez mais relacionadas com empresas, empreendimentos sociais e da filantropia na prestação de serviços de educação, que se interligam para “fazer do 'mercado' a solução óbvia para os problemas sociais e econômicos" (Ball, 2014, p. 59). Isso nos leva a conjecturar que as relações de poder e saber têm conduzido a fabricação de sujeito-professor(a)-deMatemática em conformidade com a manutenção, não só da soberania desse saber, mas de interesse do discurso econômico-capitalista.

\section{Referências}

Agamben, G. (2005). O que é um dispositivo?. Outra travessia, (5), 9-16.

Ball, D. L.; Bass, H. (2003). Making mathematics reasonable in school. In J. KILPATRICK, G. MARTIN \& D. SCHIFTER (Eds.), A Research Companion to principles and standards for school mathematics (pp. 3-14). Reston, VA: National Council of Teachers of Mathematics.

Ball, D. L.; Thames, M. H., Phelps, G. (2008). Content knowledge for teaching: what makes it special? In: Journal of Teacher Education, 59(5), 389-407.

Ball, S. J. (2014). Educação Global S.A.: novas redes políticas e o imaginário neoliberal. Ponta Grossa: UEPG.

Barbosa, J. C. (2018). Abordagens teóricas e metodológicas na Educação Matemática: aproximações e distanciamentos. In A. M. P. de Oliveira \& M. I. R. Ortigão (Orgs), Abordagens teóricas e metodológicas nas pesquisas em educação matemática (pp. 1757). Brasília: SBEM.

Barwell, R. (2013). Discursive psychology as an alternative perspective on mathematics teacher Knowledge. ZDM Mathematics Education, 45(4), 595-606.

Carrilo, J., Climent, N., Contreras, L. C., \& Munõz-Catalán, M. C. (2013). Determining Specialized Knowledge for Mathematics Teaching. In B. Ubuz, C. Haser \& M. A. Mariotti (Eds.), Proceedings VIII Congress of the European Society for Research in Mathematics Education (CERME 8) (pp. 2985-2994).

Couto, S., \& Ribeiro, M. (2017). Conhecimento interpretativo do professor que ensina matemática: o caso do cubo. Espaço Plural, 18(36), 174-195. 
DOI: $10.20396 /$ zet.v29i00.8661833

Davis, B., \& Renert, M. (2014). The Math Teachers Know: profound understanding of emergent mathematics. NY: Routledge.

Davis, B., \& Simmt, E. (2006). Mathematics-for-Teaching: an ongoing investigation of the Mathematics that teachers (need to) know. Educational Studies in Mathematics, 61, 293319.

Delaney, S. A. (2012). Validation study of the use of mathematical knowledge for teaching measures in Ireland. ZDM Mathematics Education, 44, 427-441.

Deleuze, G. (1996). Que é um dispositivo? In G. Deleuze, O mistério de Ariana (pp. 83-96). Lisboa: Vega.

Di Bernardo, R., Policastro, M. S., Almeida, A. R. de, Ribeiro, M., Melo, J. M. de, \& Aiub, M. (2018). Conhecimento matemático especializado de professores da educação infantil e anos iniciais: conexões em medidas, Cadernos Cenpec, São Paulo, 8(1), 98-124.

Di Martino P., Mellone M., \& Ribeiro M. (2019). Interpretative Knowledge. In S. Lerman (Edt), Encyclopedia of Mathematics Education. New York: Springer.

Fischer, R. M. B. (2002). A Paixão de trabalhar com Foucault. In M. V. Costa (org.), Caminhos investigativos: novos olhares na pesquisa em educação (pp. 39-60). Porto Alegre: Mediação.

Foster, C., \& Inglis, M. (2017). Teachers' appraisals of adjectives relating to mathematics tasks. Educational Studies in Mathematics, 95(3), 283-301.

Foucault, M. (1989). Microfísica do poder. $8^{\text {a }}$ Ed. Rio de Janeiro: Graal.

Foucault, M. (1999). Em defesa da sociedade: curso dado no College de France (1975-1976). São Paulo: Martíns Fontes.

Foucault, M. (2006). Ditos e Escritos V: Ética, sexualidade, política. Rio de Janeiro: Forense Universitária.

Foucault, M. (2008). Segurança, território, população: curso dado no College de France (1977-1978). São Paulo: Martíns Fontes.

Foucault, M. (2016). Arqueologia do Saber. $8^{\mathrm{a}}$ ed. Rio de Janeiro: Forense Universitária.

Foucault, M. Vigiar e Punir. Petrópolis: Vozes, 2014.

Grilo, J. S. P., Barbosa, J. C., \& Maknamara, M. (2020). Discurso da Matemática Específica para Ensinar e a Produção do Sujeito 'Professor(a)-de-Matemática'. Ciência \& Educação, Bauru, 26 (e20040).

Grilo, J. S. P., \& Barbosa, J. C. (2021). Discurso da Matemática Específica para Ensinar: a arte de governar. Educação \& Realidade, no prelo.

Hoover, M., Mosvold, R., Ball, D. L., \& Lai, Y. (2016). Making progress on mathematical knowledge for teaching. Mathematics Enthusiast, 13(1-2), 3-34.

Kim, Y. (2016). Interview Prompts to Uncover Mathematical Knowledge for Teaching: Focus on Providing Written Feedback. The Mathematics Enthusiast, 13(1), 71-92.

Kwon, M., Thames, M. H., \& Pang, J. (2012). To change or not to change: adapting mathematical knowledge for teaching (MKT) measures for use in Korea. ZDM Mathematics Education, 44, 371-385. 
DOI: $10.20396 /$ zet.v29i00.8661833

Ma, L. (1999). Knowing and teaching elementary mathematics: teachers' understanding of fundamental mathematics in China and the United States. Mahwah, NJ: Erlbaum.

Maknamara, M. (2020). Encontros entre pesquisas (auto)biográficas e necessidades de formação docente em Ciências. Revista Insignare Scientia, 3(2), 135-155.

Maknamara, M. (2011). Currículo, música e gênero: o que ensina o forró eletrônico? Tese de Doutorado em Educação. Belo Horizonte: Universidade Federal de Minas Gerais.

Menduni-Bortoloti, R., \& Barbosa, J. (2018). Matemática para o ensino do conceito de proporcionalidade a partir de um estudo do conceito. Educação Matemática Pesquisa, 20(1), 269-293.

Neubrand, M. (2018). Conceptualizations of professional knowledge for teachers of mathematics. ZDM, 50, 601-612.

Ní Ríordáin, M., Paolucci, C. \& O' Dwyer, L. M. (2017). An examination of the professional development needs of out-of-field mathematics teachers'. Teaching and Teacher Education, 64, 162-174.

Paraíso, M. A. (2014). Metodologias de pesquisa pós-críticas em educação e currículo: trajetórias, pressupostos, procedimentos e estratégias analíticas. In M. Paraíso \& D. Meyer (Orgs.), Metodologias de Pesquisas Pós-críticas em Educação. $2^{\mathrm{a}}$ ed. Belo Horizonte: Mazza.

Phelps, G., \& Howell, H. (2016). Assessing Mathematical Knowledge for Teaching: The Role of Teaching Context. TME, 13(1-2), 52-70.

Phelps, G., Kelcey, B., Liu, S. \& Jones, N. (2016). Informing Estimates of Program Effects for Studies of Mathematics Professional Development Using Teacher Content Knowledge Outcomes. Evaluation Review, 40, 383-409.

Rangel, L. G., Giraldo, V., \& Maculan Filho, N. (2015). Conhecimento de Matemática para o Ensino: Um Estudo Colaborativo sobre Números Racionais. Jornal Internacional de Estudos em Educação Matemática, 8(2), 42-70.

Ribeiro, A. J. (2012). Equação e Conhecimento Matemático para o Ensino: relações e potencialidades para a Educação Matemática. Bolema, Rio Claro (SP), 26(42B), 535558.

Ribeiro, M. (2017). Conhecimento Interpretativo para Ensinar Matemática e História da (Educação) Matemática: contributos para a Formação. Educação \& Linguagem, 20(1), 47-72.

Ribeiro, M., Mellone, M., \& Jakobsen, A. (2016). Interpreting Students Non-Standard Reasoning: Insights for Mathematics Teacher Education. For The learning of mathematics, 36(2), 8-13.

Ribeiro, M., Policastro, M. S., Almeida, A., Caldatto, M. E., Mellone, M. (2018) Conhecimento Interpretativo e especializado do professor de e que ensina matemática: uma discussão articulada em contextos de formação. Anais do VII Seminário Internacional de Pesquisa em Educação Matemática (SIPEM). Foz do Iguaçu: SBEM.

Santos, G. L. D., \& Barbosa, J. C. (2016). Um modelo teórico de matemática para o ensino do conceito de função a partir de um estudo com professores. Revista Iberoamericana de Educación Matemática, 48, 143-167. 
DOI: $10.20396 /$ zet.v29i00.8661833

Shulman, L. (1987). Knowledge and Teaching: Foundations of the new reforms. Harvard Educational Review, 57(1), 1-23.

Speer, N. M., King, K. D., \& Howell, H. (2015). Definitions of mathematical knowledge for teaching: using these constructs in research on secondary and college mathematics teachers. Journal Math Teacher Educ., 18, 105-122.

Tatoo, M. T. (ed.) (2013). Teacher Education and Development Study in Mathematics (TEDS-M): Policy, Practice, and Readiness to Teach Primary and Secondary Mathematics in 17 Countries: Technical Report. Amsterdan, IEA.

Tchoshanov, M. A. (2011). Relationship between teacher knowledge of concepts and connections, teaching practice, and student achievement in middle grades mathematics. Educational Studies in Mathematics, 76, 141-164.

Tedeschi, S. L. \& Pavan, R. (2017). A produção do conhecimento em educação: o Pósestruturalismo como potência epistemológica. Práxis Educativa, Ponta Grossa, 12(3), 116.

Veiga-Neto, A. (2016). Foucault e Educação. $3^{\mathrm{a}}$ ed. Belo Horizonte: Autêntica. 